\title{
IMPLANTAÇÃO DE SISTEMAS DE GESTÃO DA QUALIDADE DOS ALIMENTOS: REVISÃO E ANÁLISE EMPÍRICA
}

\author{
Tatiane Aparecida dos Santos Costa ${ }^{1}$ \\ Talita Costa e Silva Brito ${ }^{1}$ \\ Leandro Alberto de Azevedo ${ }^{1}$ \\ Estelamar Maria Borges Teixeira ${ }^{1}$
}

\begin{abstract}
Resumo
Esta revisão mostra as várias ferramentas utilizadas para implantação do sistema de qualidade dos alimentos em estabelecimentos do setor de alimentação. Apresentando as várias legislações que subsidia esse processo, estabelecendo critérios e instrumentos para uma correta implantação do sistema de qualidade com a finalidade de oferecer processos adequados de higiene na produção de alimentos seguros, além de favorecer a manutenção das empresas no mercado consumidor. A implantação dos sistemas de gestão da segurança de alimentos como as Boas Práticas de Fabricação (BPF), Procedimentos Operacionais Padrões (POP) e Análise de Perigos e Pontos Críticos de Controle (APPCC), a fim de garantirem a inocuidade do alimento servido e retratarem a organização da empresa. É imprescindível a implantação destes sistemas assegurando a qualidade do serviço (planejamento, controle e aperfeiçoamento). Para que sejam atingidos critérios hígidos relativos aos gêneros alimentícios, é necessária a implantação de programas de qualidade como pré-requisitos do Sistema Análise de Perigos e Pontos Críticos de Controle nos serviços de alimentação. Regulamentar e fiscalizar são apenas alguns pontos que garantem a segurança alimentar, visto que é considerado seguro o alimento que não oferece perigos à saúde do consumidor.
\end{abstract}

Palavras-chave: Segurança Alimentar; Boas Práticas de Fabricação; Qualidade dos Alimentos.

\section{Introdução}

A gestão na qualidade de alimentos é subsidiada por várias legislações com a finalidade de oferecer alimentos seguros, além de favorecer a manutenção das empresas no mercado consumidor. As Doenças Transmitidas por alimentos (DTA) encontram-se entre os riscos mais importantes em relação à saúde pública. De acordo com os dados epidemiológicos da Secretária de Vigilância em Saúde, desde 2009 até 2018 os surtos por DTA já chegaram a 6.809 notificações, com 120.584 doentes, gerando 16.632 hospitalizações e infelizmente 99 óbitos no Brasil. Não há dúvidas da importância das bactérias como agentes causadores de doenças de origem alimentar. Unidades de Alimentação e Nutrição (UAN), ou serviços de alimentação (Restaurantes entre outros) são uma das maiores fontes de surtos de doenças veiculadas por alimentos (VERGARA, 2016; ANDRADE; OLIVEIRA, 2008; SINAN, 2019).

A qualidade microbiológica dos alimentos está primeiramente relacionada, à quantidade e ao tipo de microrganismos presentes e à sua multiplicação (DUARTE, 2011). De igual modo a 
qualidade destas matérias primas, também dependem da higiene de ambientes, manipuladores e superfícies.

No Brasil, existem Legislações Federais, Estaduais e Municipais que auxiliam profissionais na atuação no serviço de alimentação, ao propiciar regulamentação dos procedimentos e normas indispensáveis na manipulação adequada de alimentos, garantindo inocuidade do alimento servido, uma vez que a utilização de manuais serve como ferramentas que retratam a organização da empresa, não podendo ser considerados completos e terminados, necessitando de uma permanente atualização, de maneira a acompanhar as alterações da empresa, sendo necessária eficiente divulgação e controle das normas nele existentes (CUNHA et al., 2013; FONSECA; SANTANA, 2012).

Segundo Tondo et al. (2015) de 1993 até 2013, foram publicadas legislações corroborando ao avanço da segurança dos alimentos em nível nacional. As indústrias alimentícias e serviços de alimentação cada vez mais estão implantando os sistemas de gestão da segurança de alimentos como as Boas Práticas de Fabricação (BPF), Procedimentos Operacionais Padrões (POP) e Análise de Perigos e Pontos Críticos de Controle (APPCC).

Apesar dos desafios para implantação destes sistemas, o sistema de Análise de Perigos e Pontos Críticos de Controle (APPCC), oferece diversos benefícios tanto para o consumidor como para a empresa, como a garantia de comercialização de alimentos seguros. Uma vez que os consumidores estão mais preocupados com os aspectos relacionados à inocuidade dos alimentos consumidos, visando evitar as Doenças Transmitidas por Alimentos (DTA) e as empresas em cumprirem as exigências pelos órgãos fiscalizadores (PADILHA et al., 2019).

De acordo com Bueno (2015) sem os programas de pré-requisitos como Boas Práticas Agrícolas, Pecuárias, de Fabricação, Manipulação e Distribuição trabalhando em conjunto, o plano do APPCC ficaria vazio.

Para garantir a segurança dos alimentos, não há dúvida que é imprescindível a implantação de sistemas de gestão da qualidade dos alimentos, como as Boas Práticas de Fabricação, dentro das unidades fornecedoras de refeições, assegurando a qualidade do serviço (planejamento, monitoramento, controle e aperfeiçoamento). O conhecimento correto e aplicação das outras ferramentas de gestão que participam deste círculo virtuoso do planejamento da qualidade como (APPCC e POP) permitem garantir as condições higiênicos sanitários dos alimentos produzidos, bem como realizar as possíveis correções, em casos de não conformidades apontadas no monitoramento. Se há registro, há evidência e consequentemente, é possível avaliar a eficácia da execução dos sistemas de gestão estabelecidos. 
O presente artigo tem como objetivo, fazer uma revisão sobre como estão os sistemas de gestão da qualidade dos alimentos no Brasil.

\section{Desenvolvimento}

De meados do século XIX até meados do século XX iniciou-se uma nova etapa histórica no campo da higiene e saúde pública, a partir dos estudos do médico francês Louis Pasteur, que foi o primeiro cientista a compreender o papel dos microrganismos nos alimentos em 1837. Impulsionando até os dias atuais um desenvolvimento extremamente rápido das pesquisas na área da alimentação (FRANCO, 2008).

As doenças causadas pela ingestão de alimentos contaminados constituem um problema mundial, apesar dos avanços tecnológicos. Segundo Andrade e Oliveira (2008) a contaminação dos alimentos ocorre por falhas na cadeia produtiva, sendo indicada pela presença de contaminantes biológicos (bactérias patogênicas e suas toxinas, vírus, parasitas e protozoários), químicos (resíduos de antibióticos, micotoxinas, pesticidas e metais pesados) e físicos (fragmentos de vidros, metais e madeiras).

De acordo com os dados epidemiológicos da Secretária de Vigilância em Saúde disponíveis na Tabela 1, somente no ano de 2018 no Brasil, ocorreram, 597 surtos de DTA notificados no Brasil, com 8.406 doentes, 916 hospitalizações e 9 óbitos relacionados (SINAN, 2019). Segundo Andrade et al. (2003) existe necessidade de avaliação microbiológicas periódicas em toda a cadeia produtiva nas unidades de alimentação e nutrição, por serem umas das maiores fontes de doenças veiculadas a alimentos no país.

Tabela 1. Perfil epidemiológico: série histórica de surtos de DTA no Brasil, 2009 a 2018.

\begin{tabular}{lllllll}
\hline Ano & Surtos & Expostos & Doentes & Hospitalizados & Óbitos & Letalidade \\
\hline 2009 & 594 & 24.014 & 9.407 & 1.328 & 12 & $0,13 \%$ \\
\hline 2010 & 498 & 23.954 & 8.628 & 1.328 & 11 & $0,13 \%$ \\
\hline 2011 & 795 & 52.640 & 17.884 & 2.907 & 4 & $0,02 \%$ \\
\hline 2012 & 863 & 42.138 & 14.670 & 1.623 & 10 & $0,07 \%$ \\
\hline 2013 & 861 & 64.340 & 17.455 & 1.893 & 8 & $0,05 \%$ \\
\hline 2014 & 863 & 124.359 & 15.700 & 2.524 & 9 & $0,06 \%$ \\
\hline 2015 & 673 & 35.826 & 10.676 & 1.453 & 17 & $0,16 \%$ \\
\hline 2016 & 538 & 200.896 & 9.935 & 1.406 & 7 & $0,07 \%$ \\
\hline 2017 & 598 & 47.409 & 9.426 & 1.439 & 12 & $0,13 \%$ \\
\hline 2018 & 597 & 57.297 & 8.406 & 916 & 9 & $0,11 \%$ \\
\hline Total & $\mathbf{6 . 8 0 9}$ & $\mathbf{6 3 4 . 5 6 8}$ & $\mathbf{1 2 0 . 5 8 4}$ & $\mathbf{1 6 . 6 3 2}$ & $\mathbf{9 9}$ & $\mathbf{0 , 0 8 \%}$ \\
\hline
\end{tabular}

Fonte: Sinan/SVS/Ministério da Saúde (2019). 
A fim de garantir a inocuidade dos alimentos, e possibilitar a coordenação de esforços no âmbito mundial e, consequentemente, consumo seguro pelos consumidores, foi criada pela Organização de Alimentação e Agricultura (FAO) e Organização Mundial da Saúde(OMS) em 1963 a Comissão do Codex Alimentarius (CAC), com o objetivo de desenvolver uma padronização dos produtos alimentícios, visando garantir a saúde dos consumidores e assegurar práticas justas no comércio de alimentos. A Comissão do Codex Alimentarius (CAC) é um organismo intergovernamental, da qual participam 185 países membros. Desde 1963 está encarregada de implementar o Programa de Padrões para Alimentos do Comitê Conjunto FAO/OMS (GERMANO; GERMANO, 2011). Os avanços normativos na área de alimentos foram observados gradualmente e, a partir da década de 1960.

O Brasil se tornou membro do Codex Alimentarius desde a década de 70. O Codex Alimentarius define higiene dos alimentos como "todas as condições e medidas necessárias para garantir a segurança e a adequação dos alimentos em todas as etapas da cadeia de alimentos" (CODEX, 2006).

De acordo com o Codex Alimentarius (2006), é necessária a implantação de programas de qualidade como pré-requisitos do Sistema Análise de Perigos e Pontos Críticos de Controle (APPCC) nos serviços de alimentação para que sejam atingidos critérios hígidos de higiene relativos aos gêneros alimentícios.

Segundo Tancredi (2004) o termo higiene aparece nas normas de alimentos como requisito dos produtos, locais onde são produzidos, manipulados ou envasados. A noção atual de higiene contempla os padrões microbiológicos, parâmetros em relação aos resíduos de pesticidas e outros contaminantes. As condições higiênicas fundamentam também a permissão de funcionamento dos locais de preparo, consumo ou comércio dos alimentos, uma vez descumprido esse preceito, e consequentemente citado nos autos de infração, pode acarretar legalmente a interdição parcial ou total, em caráter temporário, até que sejam cumpridas as exigências sanitárias de forma definitiva.

A expressão controle de qualidade refere-se à manutenção dos produtos e serviços dentro dos níveis de tolerância aceitáveis para o indivíduo (consumidor direto) ou comprador. Desse modo, deve ser mensurado o grau em que o produto satisfaz os requisitos específicos, sendo que esses níveis de tolerância e requisitos se expressam por meio de normas, padrões e especificações para avaliar a qualidade de um produto alimentício (CAVALLI; SALAY, 2001).

Para Bertolino (2010), quando se implanta um adequado sistema de controle de qualidade do alimento em todas as etapas da cadeia produtiva, se promove também a motivação profissional, e de 
igual modo se estimula a atualização constante e a elevação profissional das categorias envolvidas. Caracterizam-se como atividades de controle da qualidade as análises físico-químicas, sensoriais e microbiológicas; técnicas e atividades operacionais usadas para atender os requisitos para a qualidade; avaliação de insumos, matérias-primas e embalagens; tarefa de execução do controle do produto em processo; avaliação dos requisitos de higiene; e atendimento de especificações para o produto final.

Os requisitos de higiene são citados no Decreto-lei no 986/1969, vigente, como parte dos Padrões de Identidade e Qualidade (PIQ) para cada tipo ou espécie de alimento, e compreendem as medidas sanitárias concretas e demais disposições necessárias à obtenção de um alimento seguro. Posteriormente, em 1993, a portaria $\mathrm{n}^{\circ} 1.428$, estabeleceu os critérios para os padrões de identidade e qualidade de produtos e serviços relacionados ao controle dos alimentos em todo o território nacional brasileiro. Atualmente se configuram com as boas práticas de fabricação e manipulação de alimentos (BRASIL, 1969; BRASIL, 1993).

Visando melhorar as condições higiênico-sanitárias na preparação de alimentos e adequar as ações da Vigilância Sanitária, o Ministério da Saúde publicou a Portaria no 326/1997, definindo as condições técnicas para elaboração do Manual de Boas Práticas (BRASIL, 1997). Conjuntamente com as boas práticas, a ANVISA, através da Resolução RDC n ${ }^{0}$ 275/2002, definiu o Regulamento Técnico de Procedimentos Operacionais Padronizados (POP), aplicados aos estabelecimentos produtores de alimentos nas seguintes situações: higienização das instalações, equipamentos, móveis e utensílios; controle da potabilidade da água; higiene e saúde dos manipuladores; manejo dos resíduos; manutenção preventiva e calibração de equipamentos; controle integrado de vetores e pragas urbanas; e programa de recolhimento de alimentos (BRASIL, 2002).

A Agência Nacional de Vigilância Sanitária (ANVISA) também publicou a RDC ${ }^{\circ}$ 216/2004, que abrange os procedimentos que devem ser adotados nos serviços de alimentação, a fim de garantir as condições higiênico-sanitárias do alimento preparado (BRASIL, 2004).

Regulamentar e fiscalizar são alguns dos pontos que garantem uma melhor qualidade dos alimentos preparados. Sendo fundamental para alcançar este objetivo, os Serviços de Alimentação assumam um papel protagonista, sendo responsáveis por práticas e ambientes adequados para a manipulação de alimentos (MEDEIROS et al., 2012).

Assis (2012) define segurança alimentar como acesso de todas as pessoas a uma alimentação nutricionalmente adequada, suficiente e segura. A Segurança Alimentar é um conceito construído ao longo das últimas décadas do século XX, pelo confronto do problema da fome que demonstrava 
a necessidade de ações mais amplas para o enfrentamento desse flagelo da humanidade (ORTEGA; BORGES, 2012).

A Organização de Alimentação e Agricultura (FAO) define segurança alimentar (food security) como "a situação na qual toda população tem pleno acesso físico e econômico a alimentos seguros e nutritivos que satisfaçam as suas necessidades e preferências nutricionais, para levar uma vida ativa e saudável" (FAO, 2009).

A realização do direito de todos ao acesso regular e permanente a alimentos de qualidade, em quantidade suficiente, sem comprometer o acesso a outras necessidades essenciais, tendo como base práticas alimentares promotoras de saúde que respeitem a diversidade cultural e que sejam ambiental, econômica e socialmente sustentáveis, também definem a segurança alimentar segundo a Lei Orgânica de Segurança Alimentar e Nutricional - Lei nº 11.346/2006 (BRASIL, 2006).

Os sistemas de gestão da qualidade e segurança de alimentos são importantes métodos de monitoramento da linha de produção, e quando aplicados, garantem sucesso em vários serviços de alimentação em todo o mundo. Para a garantia do cumprimento aos requisitos de segurança alimentar, o planejamento de produtos e processos, algumas das ferramentas disponíveis são a implementação das BPF (Boas Práticas de Fabricação); BPA (Boas Práticas Agrícolas); implantação do sistema APPCC (Análise de Perigos e Pontos Críticos de Controle); e a ISO 22000, entre outras (FORSYTHE, 2013; VERGARA, 2016).

Entre as normas ISO relacionadas à área de alimentos, vale destacar a ISO 22000, por ser o primeiro padrão internacional para implementação de um sistema de gestão de segurança alimentar. A ISO 22000 apresenta como elementos-chave: Sistema de Gestão da Qualidade; Programa de PréRequisitos (Boas Práticas), Controles de Perigos através do Sistema APPCC e Comunicação Interativa ao longo de toda cadeia alimentar (SCHILLING, 2008; ABNT, 2006).

A Organização Internacional para a Normatização (ISO) foi fundada em 1947 em Genebra, Suíça, e sua missão é promover a normatização de produtos e serviços para que a qualidade deles seja sempre aperfeiçoada. A expressão ISO tem o significado semântico de igualdade ou padrão, sendo representada em vários países por organismos locais que seguem os procedimentos definidos por esse comitê. No Brasil, a organização responsável pela adaptação aos padrões ISO em diversas atividades empresariais é a Associação Brasileira de Normas Técnicas (ABNT) (ROCHA et al., 2009).

A ABNT publicou, em 2008, a NBR 15635, que estabelece os requisitos de boas práticas higiênico-sanitárias e controles operacionais essenciais a serem seguidos por estabelecimentos que 
desejem comprovar e documentar que produzem alimentos em condições higiênico-sanitárias adequadas para o consumo (ABNT, 2008).

Segundo Germano e Germano (2011), alimento seguro é aquele que não oferece perigos à saúde do consumidor, sejam de origem biológica, química ou física. Tais perigos devem ser controlados para que não comprometa a qualidade do alimento. Segundo o autor, o conceito de inocuidade dos alimentos inclui aspectos que vão desde os locais onde são produzidos alimentos de origem animal e vegetal para consumo, e seus subprodutos, até chegar à mesa do consumidor. Nesse processo, todos são responsáveis pela manutenção da inocuidade dos alimentos, evitando que estes se transformem em fonte de doenças, incluindo as autoridades governamentais, produtores agropecuários, transportadores de matéria-prima e produtos industrializados, indústrias processadores, atacadistas, varejistas, universidades, empresas de comunicação social entre outros, e o consumidor final.

De acordo com Marins, Tancredi (2014), o princípio da precaução, igualmente indispensável na atualização do direito dos consumidores, surgiu com a globalização e os riscos aumentados, por envolver importante área do direito que diz respeito à responsabilidade dos produtores de alimentos. Dessa forma, os conceitos e entendimentos atuais sobre higiene, saúde pública, qualidade, inocuidade e segurança alimentar, foram consideravelmente ampliados, com o objetivo de estabelecer uma relação harmônica entre a integridade; capacidade de desenvolvimento; e higidez necessária à manutenção da vida. Sendo claro que, dependem ainda da ingestão diária de alimentos quantitativa e qualitativamente adequados, de modo a não oferecer risco à saúde do consumidor.

Atualmente, uma das mudanças percebidas no perfil do consumidor é o aumento da procura pelos serviços de alimentação seguros. Essa crescente demanda junto ao maior grau de exigência dos consumidores em busca de qualidade faz com que empresários se aprimorem e busquem diferentes formas de apresentação de seus empreendimentos (CARVALHO et al., 2012).

$\mathrm{Na}$ indústria alimentícia a gestão da qualidade enfoca a aplicação de métodos que visem avaliar a qualidade percebida pelo consumidor e identificar a qualidade que ele espera de um produto, uma vez que propriedades sensoriais (cor, cheiro, sabor, textura, viscosidade etc.), a forma de apresentação do produto e ausência de perigos são aspectos priorizados na escolha do alimento (SANTOS; ANTONELLI, 2011).

A garantia da segurança dos alimentos por meio das Boas Práticas de Fabricação (BPF) e do sistema de Análise de Perigos e Pontos Críticos de Controle (APPCC) ou norma ISO são prérequisitos para se ter um gerenciamento efetivo da qualidade. Tendo sempre como foco, à produção 
de um alimento seguro que não ofereça riscos à saúde do consumidor, buscando continuamente a melhoria dos produtos e processos (GRIGG; MCALINDEN, 2001; VERGARA, 2016).

\section{Análise empírica}

Os estudos mostram a maior eficiência quando há conciliação de várias ferramentas de gestão do controle de qualidade. Apesar de extensivos ensinamentos sobre higiene dos alimentos, para a prevenção de doenças de origem alimentar, a incidência de surtos e casos esporádicos continuam a acontecer no Brasil. Segundo Biedrzycki et al. (2011), a utilização de determinados métodos sozinhos no controle da qualidade dos alimentos nos serviços de alimentação, como as Boas Práticas Fabricação (BPF), muitas vezes consegue atingir os padrões mínimos de qualidade sanitária, porém não são suficientes para a perfeita segurança dos alimentos. Sob o mesmo ponto de vista, outros autores concluem que as BPF sozinhas não conseguem combater as DTA. Outros concordam que as legislações como a RDC n 216 e algumas portarias, além da criação de manual de BPF, facilitam o alcance de melhores resultados na qualidade e nos processos de gestão.

E corroborando com os estudos de Forsythe (2013) o APPCC constitui um importante método preventivo empregado na linha de produção em serviço de alimentação. Verificamos que quando implantado em locais onde não há eficiência dos pré-requisitos ou ausência desses, os resultados não são satisfatórios. Isso mostra a importância da correta aplicação e verificação cíclica dos programas considerados mais básicos e assim obter as vantagens da aplicação da ferramenta.

O sistema APPCC tem um resultado muito positivo, principalmente quando comparado com outras ferramentas de controle de qualidade. Com tudo fica evidente nos estudos, que a implantação do APPCC, além dos benefícios relativos a qualidades, está claramente relacionada a uma significativa redução dos custos de produção. Isso deve-se pela necessidade de ter juntamente com ele as boas práticas de fabricação e procedimentos operacionais padronizados (POP), que são prérequisitos para o sistema, além de ser feito especificamente para cada produto e/ou procedimento. Outro ponto relevante para alcançar melhores resultados, diz respeito à união de todas estas ferramentas às equipes, mostrando o porquê e como cada um destes programas são importantes durante o processo para se garantir um produto seguro. A percepção desse equilíbrio varia em função da heterogeneidade dos agentes produtivos envolvidos na implementação e desenvolvimento do sistema APPCC.

Entre as principais motivações relatadas pelos representantes das organizações enfocadas, predominam a busca de desenvolvimento das funções de política e estratégia de qualidade, de 
maneira a alinhar os objetivos do sistema APPCC, com os objetivos e estratégias da empresa, e das funções de garantia de qualidade, de maneira a assegurar para clientes e consumidores, que as demandas de qualidade sejam atendidas. Segundo Biedrzycki et al. (2011) para a exportação o APPCC se torna obrigatório, por ser um programa que é mandatário na questão de segurança alimentar, sendo esse o principal motivo de implantação.

Um dos indicadores de desempenho do sistema APPCC mais citado é relacionado à diminuição do percentual de reclamações de clientes e consumidores vinculadas à qualidade do produto final. Concomitantemente para que isto ocorra, a avaliação de não-conformidades e desvios nos padrões microbiológicos nos pontos críticos de controle (PCC), no processo e nos produtos acabados, são conclusivos para o sucesso dos indicadores de desempenho do sistema APPCC.

As principais dificuldades relacionadas à manutenção do sistema APPCC residem no manejo da documentação, como o tempo demandado e capacitação dos responsáveis, comprometimento dos funcionários no correto preenchimento dos registros e na percepção dos dados que realmente necessitam ser monitorados e registrados (BIEDRZYCKI et al., 2011). Para muitos dos pesquisadores, manter o programa funcionando é mais complicado do que implementar, normalmente porque as cozinhas industriais ou serviços de alimentação trabalham com número de colaboradores no limite, e uma vez instituído o APPCC, demandará em novas contratações. A cadeia produtiva de uma unidade de alimentação ou serviço de alimentação é complexa, sendo necessário um gestor compreender que muito além de se produzir e servir, é preciso garantir inocuidade em toda a cadeia produtiva (linha quente e fria da produção). Para isto os registros de controles de recebimento, estocagem, manipulação, higienização, sanitização, pré-preparo, cocção, temperaturas, amostras 72 horas, e a distribuição, são indispensáveis para garantir uma excelente gestão da qualidade do alimento produzido. Bem como os registros da saúde dos manipuladores, de acordo com as legislações vigentes.

O controle da qualidade dos alimentos deve ser praticado de forma contínua, e não apenas no produto final, com vias a oferecer maior garantia aos usuários do serviço, o que viabiliza aumento da confiabilidade por parte dos consumidores e minimização dos riscos à saúde. Sendo de responsabilidade do prestador de serviço selecionar fornecedores, realizando visitas técnicas em seus estabelecimentos, garantindo que o alimento chegue em condições higiênicas seguras para ser manipulado e/ou produzido dentro do serviço de alimentação. Realizando também um processo seletivo capaz de selecionar os funcionários com base em critérios éticos e legais, bem como os qualificar nas legislações vigentes a alimentos, e posteriormente, garantir que seja estabelecido 
diariamente o aprimoramento das atividades executadas, de forma a ampliar a confiança e a responsabilidade na manipulação adequada dos alimentos, garantir a segurança higiênico-sanitária do alimento produzido, além de possibilitar a redução dos custos para a empresa.

Segundo Borges (2017) a falta de controle e acompanhamento dos processos de produção e manipulação de alimentos, são os principais fatores do baixo índice de conformidade nos serviços de alimentação. Realmente a implantação de Boas Práticas de Fabricação (BPF), apesar de sozinha não serem totalmente eficiente, ainda assim trazem resultados positivos nos quesitos relacionados à segurança dos alimentos, uma vez que engloba o cuidado em ensinar aos manipuladores a importância da qualidade dos alimentos e do serviço que estão prestando como manipuladores de alimentos, conscientizando-os que precisam servir um alimento seguro. Boas Práticas vão além do cuidado com a fabricação, estão relacionadas diretamente com as boas práticas de higiene, de comportamento que são importantíssimas para manter satisfatórias as condições higiênico-sanitárias do estabelecimento. O checklist é uma ferramenta importante, pois garante que todos os processos sejam monitorados e as evidências sejam estabelecidas. Outra ferramenta complementar que esta crescente no mercado atual, é a utilização de consultorias em serviços de alimentação, pois apresentam resultados positivos para o crescimento da empresa, quando comparados com aqueles que não buscam esse serviço ou não têm profissionais devidamente capacitados e responsáveis técnicos. Quando aplicada, são notórios a melhoria e o crescimento da empresa em estar de acordo com os padrões legais de higiene e qualidade dos itens analisados.

\section{Conclusão}

As legislações são importantes suportes para o alcance de resultados satisfatórios, estabelecendo critérios e instrumentos para uma correta avaliação dos processos de higiene na produção de alimentos. Por isto, a gestão da qualidade dos alimentos dentro de serviços de alimentação é importantíssima, pois otimiza o serviço, reduz o desperdício, e principalmente, garante alimentos livres de contaminantes e com valor nutricional agregado, além de trazer benefícios a saúde do consumidor e reduzir internações hospitalares por intoxicações alimentares. O reflexo de uma boa gestão da qualidade está na satisfação e fidelização dos consumidores ao produto/alimento servido, bem como na confiança aos serviços prestados pela empresa. Quando se implanta um adequado sistema de controle de qualidade nas etapas de processamento do alimento, também se promove a motivação profissional e se estimula a atualização constante e a elevação profissional das categorias envolvidas. 


\section{Referências}

ANDRADE, N. J. et al. Avaliação das condições microbiológicas em unidades de alimentação e nutrição. Rev. Ciênc. Agrotec., Lavras. V. 27, n. 3, p. 590-596, maio/jun, 2003.

ANDRADE, N. J.; OLIVEIRA P. C. L. Higienização na indústria de alimentos e segurança alimentar. In: BASTOS, Maria do Socorro Rocha (org.). Ferramentas da ciência e tecnologia para a segurança dos alimentos. Fortaleza: Embrapa Agroindustrial Tropical, 2008. p. 41-66.

ASSIS, L. Alimentos Seguros: Ferramentas para a gestão e controle da produção e distribuição. $1^{\text {a }}$ reimpressão. Rio de Janeiro: Senac Nacional, 2012. 360 p.

ASSOCIAÇÃO BRASILEIRA DE NORMAS TÉCNICAS (ABNT). NBR ISO 15635: Serviço de alimentação - Requisitos de boas práticas higiênico-sanitárias e controles operacionais essenciais. Rio de Janeiro: ABNT, 2008.

ASSOCIAÇÃO BRASILEIRA DE NORMAS TÉCNICAS (ABNT). NBR ISO 22000 - Sistema de Gestão da Segurança de alimentos - Requisitos para qualquer organização na cadeira produtiva de alimentos. Rio de Janeiro: ABNT, 2006.

BIEDRZYCKI. A. et al. Estudo do processo de implementação do sistema APPCC em empresas processadoras de lácteos. Rev. Inst. Latic. "Cândido Tostes", Santa Terezinha, v. 66, n. 379, p. 35:44, Mar/Abr, 2011.

BERTOLINO, M. T. Gerenciamento da qualidade na indústria alimentícia, com ênfase na segurança dos alimentos. Porto Alegre: Artmed, 2010. 320 p.

BORGES, E. V. A. Relato de experiência: Diagnóstico e implantação das boas práticas em estabelecimentos na cidade de Unaí -MG. Dissertação apresentada ao programa de Pós-graduação em Ciência e Tecnologia de Alimentos. IFTM Campus Uberaba, 2017.

BRASIL. Decreto-lei no 986, de 21 de outubro de 1969. Dispõe sobre normas básicas sobre alimentos dos Ministérios da Marinha de Guerra, do Exército e da Aeronáutica Militar. Diário Oficial da União, Brasília, seção 1, p. 8.935, 21 out. 1969.

BRASIL. Ministério da Saúde. Agência Nacional de Vigilância Sanitária. Portaria no 1.428, de 26 de novembro de 1993. Regulamento Técnico para Inspeção Sanitária de Alimentos, estabelecimentos de padrões de identidade e qualidade na área de alimentos e serviços e regulamento técnico de Boas Práticas de Produção e Prestação de Serviços na área de Alimentos. Diário Oficial da União, Brasília, DF, 02/12/1993.

BRASIL, Ministério da Saúde. SVS. Portaria no 326 de 30 de julho de 1997. Aprova o Regulamento Técnico; "Condições Higiênico-Sanitárias e de Boas Práticas de Fabricação para Estabelecimentos Produtores/Industrializadores de Alimentos". Diário Oficial da União; Brasília,01 de agosto de 1997, Seção 1.

BRASIL, Ministério da Saúde, Agência nacional de Vigilância Sanitária. Resolução RDC n 275 , de 21 de outubro de 2002. Dispõe sobre o Regulamento Técnico de Procedimentos Operacionais Padronizados aplicados aos Estabelecimentos Produtores/Industrializadores de Alimentos e a Lista de Verificação das Boas Práticas de Fabricação em Estabelecimentos Produtores/Industrializadores de Alimentos. Diário Oficial da União; Brasília, DF, 23 de outubro de 2002, seção 1, p. 126. 
BRASIL. Ministério da Saúde. Agência Nacional de Vigilância Sanitária. Resolução RDC n . 216, de 15 de setembro de 2004 b. Dispõe sobre regulamento técnico de boas práticas para serviços de alimentação. Diário Oficial da União, Brasília, DF, 16 de set. de 2004, Seção 1, p. 25.

BRASIL, Ministério do Desenvolvimento Social e Combate à Fome. Lei no 11.346 , de 15 de setembro de 2006. Cria o Sistema Nacional de Segurança Alimentar e Nutricional - SISAN com vistas em assegurar o direito humano à alimentação adequada. Diário Oficial da União; Brasília, 18 de setembro de 2006, p. 1.

BUENO, M. C. Ferramentas da qualidade. Os 7 princípios para implementar o sistema de análise de perigos e pontos críticos de controle (APPCC). 2015. Disponível em:

$<$ https://blogdaqualidade.com.br/os-7-principios-para-implementar-o-sistema-de-analise-deperigos-e-pontos-criticos-de-controle-appcc/\#> Acesso em 24 de maio de 2020.

CARVALHO, R. D. et al. A conduta dos consumidores em restaurante self service institucional correlacionada à segurança dos alimentos. Enciclopédia Biosfera, Centro Científico Conhecer, Goiânia, v.8, n.15; p. 2321- 2333, novembro, 2012.

CAVALLI, S. B.; SALAY, E. Segurança do alimento e recursos humanos: estudo exploratório em restaurantes comerciais dos municípios de Campinas, SP e Porto Alegre, RS. Higiene Alimentar, São Paulo, v. 18, n. 126-127, p. 29-35, nov/dez. 2001.

CODEX Alimentarius. Higiene dos alimentos: textos básicos. Brasília: Organização PanAmericana da Saúde, 2006.

CUNHA, F. M. F. et al. Desafios da gestão da segurança dos alimentos em unidades de alimentação e nutrição no Brasil: uma revisão. Rev. Contextos, São Paulo, v. 1, n. 2, p.4-14, março, 2013.

DUARTE, E. R. Microbiologia Básica para Ciências Agrárias/Eduardo Robson Duarte, (ed.). Montes Claros: Instituto de Ciências Agrárias da UFMG, 2011.

FAO/WHO (2009). CODEX ALIMENTARIUS. Internacional Food Standards. Food and Agriculture Organization of the United Nations \& World Health Organization. Disponível em: http://www.codexalimentarius.net/web/index_en.jsp. Acesso: 26/05/2020.

FAO/WHO (2014). CODEX ALIMENTARIUS. Internacional Food Standards. Food and Agriculture Organization of the United Nations \& World Health Organization. Disponível em: http://www.codexalimentarius.org/about-codex/en/. Acesso: 26/05/2020.

FONSECA, K. Z; SANTANA, G. R. Guia prático para gerenciamento de unidades de alimentação e nutrição. Cruz das Almas. BA. UFRB, 2012.

FORSYTHE, S. J. Microbiologia da Segurança dos Alimentos. 2. ed. Porto alegre: Artmed, 2013. $607 \mathrm{p}$.

FRANCO, B. D. G. M. Importância dos microrganismos nos alimentos. In: LANDGRAF, M. Microbiologia dos alimentos. São Paulo: Atheneu, 2008. p. 1-12.

GERMANO, P. M. L.; GERMANO, M. I. S. Higiene e vigilância sanitária de alimentos. 4. ed. Barueri: Manole, 2011. P. 631.

GRIGG, N.; MCALINDEN, C. A new role for ISO 9000 in the food industry? Indicative data from the UK and mainland Europe. British Food Journal, Reino Unido, v. 103, n. 9, p. 644-656, 
outubro, 2001.

MARINS, B. R.; TANCREDI, R. C. P. Segurança alimentar no contexto da vigilância sanitária: reflexões e práticas. Escola Politécnica de Saúde Joaquim Venâncio. Rio de Janeiro, 2014. 288 p.

MEDEIROS, L. B. et al. Diagnóstico das condições higiênicas de serviços de alimentação de acordo com a NBR 15635:2008. Brazilian Journal Food Technology, Campinas, vol.15, n. spe, p. 47-52, dezembro, 2012.

ORTEGA, A. C.; BORGES, M. S. Codex Alimentarius: a segurança alimentar sob a ótica da qualidade. Segurança Alimentar e Nutricional, Campinas, v.19, nº 1, p. 71-81, fevereiro, 2012.

PADILHA. M. R. F. et al. Benefícios e desafios da implantação de APPCC em indústrias de alimentos para segurança de alimentos. Contextos da Alimentação - Revista de Comportamento, Cultura e Sociedade Vol. 7, n. 2, 2019. São Paulo: Centro Universitário Senac.

ROCHA, J. M. et al. ISO 22000: Gestão da Segurança dos Alimentos. Revista ADMpg Gestão Estratégica, Ponta Grossa, v.2, n.2, p. 59-66, 2009.

SANTOS, A. B.; ANTONELLI, S. C. Aplicação da abordagem estatística no contexto da gestão da qualidade: um survey com indústrias de alimentos de São Paulo. Gest. Prod., São Carlos, v. 18, n. 3, p. 509-524, junho, 2011.

SCHILLING, M. Qualidade em Nutrição. São Paulo: Livraria Varela, 2008. 248p.

SINAN. Ministério da Saúde. Secretária de Vigilância em Saúde. Departamento de Vigilância das Doenças Transmissíveis. Surtos de Doenças Transmitidas por Alimentos no Brasil. Coordenação Geral de Doenças Transmissíveis. Informe 2018, p. 6, fev. de 2019. Disponível em:

$<$ https://portalarquivos2.saude.gov.br/images/pdf/2019/fevereiro/15/Apresenta----o-Surtos-DTA--Fevereiro-2019.pdf $>$ Acesso em 27 de maio de 2020.

TANCREDI, R. C. P. et al. Vigilância sanitária municipal do Rio de Janeiro: considerações sobre a aplicabilidade normativa no controle de alimentos. Revista de Direito Sanitário, São Paulo, v. 5, n. 3, p. 85-98, nov. 2004.

TONDO, E. C. et al. Avanços da segurança de alimentos no Brasil. Vigilância Sanitária em Debate, Rio de Janeiro, v. 3, n. 2, p. 122-130, 2015. Disponível em:

$<$ https://www.researchgate.net/publication/276511032_Avancos_da_seguranca_de_alimentos_no_ Brasil $>$ Acesso em: 24 de maio de 2020.

VERGARA, C. M. A. C. Gestão da qualidade na área de alimentos. Nutrivisa - Revista de Nutrição e Vigilância em Saúde, Fortaleza, v. 2, n. 3, p. 99;100, novembro/2015-fevereiro/2016. Disponível em <https://www.revistanutrivisa.com.br/wp-content/uploads/2016/03/nutrivisa-vol-2num-3-b.pdf>. Acesso em 27/05/2020.

\title{
IMPLEMENTATION OF FOOD QUALITY MANAGEMENT SYSTEMS: REVIEW AND EMPIRICAL ANALYSIS
}

\begin{abstract}
This review shows the various tools used to implemente the food quality system in food


establishments. Presenting the various laws that subsidize this process, establishing criteria and instrumentes for a correct implementation of the quality system in order to offer adequate hygiene processes in the production of safe food, in addition to favoring the maintenance of companies in the consumer market. The implementation of food safety management systems such as Good Manufacturing Practices (GMP), Standard Operating Procedures (SOP) e Hazard Analysis and Critical Control Points (HACCP), in order to guarantee the safety of the food served and portray the organization of the company. It is essential to implement these systems ensuring the quality of the servisse (planning, control and improvement). In order to achieve healthy criteria related to foodstuffs, it is necessary to implement quality programs as prerequisites of the Hazard Analysis System and Critical Control Points in food services. Regulating and inspecting are just a few points that guarantee food security, since food that does not pose a health hazard to consumers is considered safe.

Keywords: Food Security; Good Manufacturing Practices; Food Quality.

\title{
IMPLEMENTACIÓN DE SISTEMAS DE GESTIÓN DE LA CALIDAD DE LOS ALIMENTOS: REVISIÓN Y ANÁLISIS EMPÍRICO
}

\begin{abstract}
Resumen
Esta revisión muestra las diversas herramientas utilizadas para implementar el sistema de calidad de los alimentos in establecimientos de alimentos. Presentando las diversas leyes que subsidian este proceso, estableciendo criterios e instrumentos para una correcta implementación del sistema de calidad con el fin de ofrecer procesos adecuados de higiene en la producción de alimentos seguros, además de favorecer el mantenimiento de las empresas en el mercado de consumo. La implementación de sistemas de gestión de inocuidad de los alimentos, como las Buenas Prácticas de Fabricación (BPF). Estándar de Procedimientos Operativos (POP) y análisis de peligros y puntos críticos de control (APPCC) para garantizar la seguridad de los alimentos servidos y retratar la organización de la empresa. Es esencial implementar estos sistemas asegurando la calidad del servicio (planificación, control y mejora). Para lograr criterios saludables relacionados con los alimentos, es necesario implementar programas de calidad como requisitos previos del Sistema de Análisis de Peligros y Puntos Críticos de Control en los servicios de alimentos. La regulación y la inspección son solo algunos puntos que garantizan la seguridad alimentaria, ya que los alimentos que no representan un peligro para la salud de los consumidores se consideran seguros.
\end{abstract}

Palabras clave: Seguridad alimentaria; Buenas prácticas de fabricación; Calidad de la comida.

\author{
Agradecimentos \\ Agradecemos ao Instituto Federal do Triângulo Mineiro - IFTM Campus Uberaba por ministrar a \\ disciplina de Segurança Alimentar e Gestão da Segurança dos Alimentos no curso de Mestrado \\ Profissional em Ciências e Tecnologia dos Alimentos.
}

